\title{
SUMMARY PROGRESS REPORT ON
}

\section{THERMAL PLASMA PROCESSING OF MATERIALS}

\author{
prepared for \\ Dr. Oscar P. Manley \\ Division of Engineering \& Geosciences \\ Office of Basic Energy Sciences, ER-15 \\ Departmeni of Energy \\ prepared by \\ Profs. E. Pfender and J, Heberlein \\ Department of Mechanical Engineering \\ University of Minnesota \\ Minneapolis, MN. 55455
}

Grant Number: DOE-FG02-85ER-13433

Period:

9-1-1988 to 1-31-1992

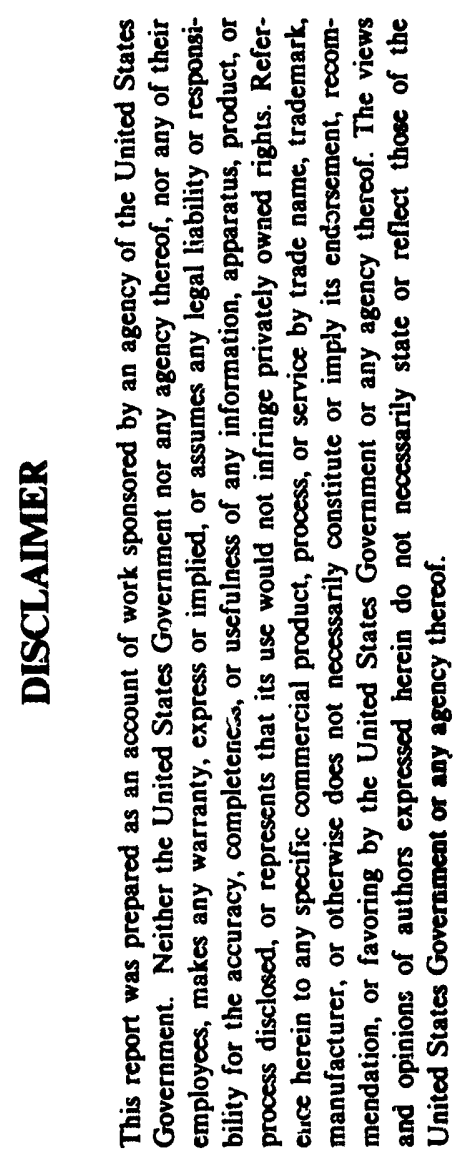

February 1992 


\section{TABLE OF CONTENTS}

\section{Page}

1. Introduction 1

2. Studies of Plasma Jet Behavior 2

3. Injection of Particulates into a Thermal Plasma $\quad 7$

4. Plasma Reactors for Plasma Synthesis \& Plasma CVD 8

5. Synthesis of Fine Powders 10

6. Plasma Chemical Vapor Deposition 14

$\begin{array}{lll}\text { 7. References } & 16\end{array}$

8. Publications (papers published during the reporting period)
a. Journal Publications
b. Other (Proceedings) 


\section{INTRODUCTION}

- During the reporting period, emphasis of our work has been on plasma synthesis of fine powders, plasma Chemical Vapor Deposition (CVD), on related diagnostics, and on modeling work.

Since plasma synthesis as well as plasma CVD make frequent use of plasma jets, the beginning of the reporting period has been devoted to studies of the properties and characteristics of plasma jets and the behavior of particulates injected into such plasma jets.

Although most of the construction of the Triple-Torch Plasma Reactor (TTPR) preceded this reporting period, a number of modifications have been made in particular modifications required for plasma CVD of diamond. A new reactor designed for Counter-Flow Liquid Injection Plasma Synthesis (CF LIPS) proved to be an excellent tool for synthesis of fine powders as well as for plasma CVD. An attempt was made to model flow and temperature fields in this reactor. Substantial efforts were made to single out those parameters which govern particle size, size distribution, and powder quality in our plasma synthesis experiments. This knowledge is crucial for controlling the process and for meaningful diagnostics and modeling work. Plasma CVD of diamond films, using both reactors has been very successful and we have been approached by a number of companies interested in using this technology for coating of tools. 


\section{STUDIES OF PLASMA JET BEHAVIOR}

Commercial plasma torches produce usually strongly turbulent plasma jets. The flow structure of such a plasma jet has been considered operated with argon as plasma gas in an air environment [1]. Conditional mean velocity and turbulence intensity measurements were made using laser doppler anemometry. Velocity measurements conditional on fluid originating from the plasma jet and from the entrained air were obtained by alternately seeding only the plasma gas or the surrounding air. These results strongly imply that an engulfment-type entrainment mechanism takes place in turbulent plasma jets as opposed to a simple diffusion process. This finding has been further corroborated by using additional diagnostic techniques, including coherent Anti-Stokes Raman Spectroscopy (CARS) (in cooperation with the Idaho National Engineering Laboratories), emission spectroscopy, and enthalpy and sampling probe techniques [2]. When the information obtained from these individual techniques is combined, it is possible to put together a picture of the large-scale flow structures which govern the entrainment process in a circular plasma jet and how these structures break down and lead to the onset of turbulence. A qualitative picture of this situation is shown in Fig. 1.

As the plasma jet exits the nozzle, it forms a steep laminar shear layer at the outer edge of the jet as shown in Fig. 1. This large velocity difference causes the flow around the nozzle exit to roll up into a ring vortex which is pulled downstream with the flow, allowing the process to repeat itself again at the nozzle exit. Adjacent vortex rings on the outer edge of the jet have the tendency to coalesce, forming larger vortices; perturbations to these vortices then cause a wave instability to start growing around the entire ring. Next, 
the distorted vortex rings start entangling themselves with adjacent rings, finally resulting in total breakdown of the vortex structure into large-scale eddies and the onset of turbulent flow. It is the entanglement process of adjacent unstable vortices that results in the first large-scale engulfment or entrainment of the external air, although some entrainment also takes place during the roll-up process of the jet's shear layer. These large eddies of cold air that are entrained have a much higher density and thus greater inertia than their high temperature counterparts. The eddies of air travel in the axial direction at much lower velocity while the hot plasma gas essentially accelerates around and stagnates on the cold air eddies with little initial mixing. All eddies in the flow are continually breaking down into smaller and smaller eddies, while diffusion is taking place on the molecular level at all eddy boundaries. This whole process represents an excellent example of continually increasing entropy in a system. The mixing and diffusion process eventually reaches the center line of the jet, foretelling the end of the potential core. The jet now undergoes transition and eventually becomes fully turbulent while eddies of external air continue to be engulfed and then absorbed into the main jet along its entire length, further reducing both the mean temperature and the velocity.

The high entrainment rate of external air has been confirmed by shadowgraphs and by CARS measurements [2] as well as by probe measurements [3] using an oxygen analyzer and a gas chromatograph for determining gas composition in the plasma jet. By $30 \mathrm{~mm}$ downstream the jet is only $50 \%$ argon on the centerline and overall the jet is probably less than one-third argon. 
The high noise levels associated with turbulent plasma jets, considered to be a direct result of large-scale coherent structures forming and breaking down, provide further evidence that these structures exist.

There are, however, significant differences between plasma jets operated in ambient air and those operated in a controlled atmosphere, as for example, in argon [4]. Although the local specific enthalpies are very similar in ambient air and in ambient argon, the densities and specific heat differ substantially. Consequently the volumetric content of energy is much lower in the case of pure argon. This is reflected by the differences in temperatures, which are found to be 50 to $60 \%$ higher in the case of pure argon.

Velocities measured in pure argon exceed the values measured in ambient air by approximately $20 \%$ in the downstream region of the jet and up to $50 \%$ close to the nozzle exit.

A striking difference is noticed in the shape and size of the two tail flames. The pure argon plasma jet appears wider and longer with betterdefined and more regular boundaries compared to its counterpart in ambient air.

The explanation for the different appearance lies in the plasma composition of these jets. It has been shown [3] that the air entrained into the jet can account for as much as $\mathbf{8 0 \%}$ of the total concentration in the fringes and up to $30 \%$ in the center at locations close to the nozzle exit. With increasing temperature, oxygen will first dissociate (around $4000 \mathrm{~K}$ ), followed by nitrogen (around $7000 \mathrm{~K}$ ), resulting in a substantial increase in the specific heat of the plasma, reaching values one order of magnitude higher than in the case of pure argon. 
These differences in the specific heat are responsible for the higher temperatures found in the case of pure argon plasma jets, which therefore are able to extend the plasma further axially as well as radially.

The same phenomenon (dissociation) gives rise to a strong exchange of energy between the jet periphery and its core. As the nitrogen and oxygen molecules enter the hot jet, due to turbulent mixing and diffusion, they dissociate and the atoms diffuse toward the fringes, driven by concentration gradients. Since there is no net mass flux, the molecules in the fringes diffuse toward the center of the plasma where they dissociate, i.e. there is a net transport of dissociation energy from the hotter part of the plasma jet to the fringes and the surroundings.

Compared to the operation in pure argon, it seems that the plasma jet in ambient air experiences an earlier transition to turbulence, much closer to the nozzle exit. This is confirmed by a comparison of local Reynolds numbers, which in the case of a plasma jet in ambient air are always much higher (at least 100\%) than those in pure argon, because of the lower temperatures and the corresponding higher densities and lower viscosities.

Due to the complex nature of the previously described turbulent plasma jets, modeling of such jets remains a formidable task. Numerical work is currently incapable of modeling the real process of entrainment with a succession of hot and cold eddies of flow just outside of the potential core region, the breakdown of eddies and the mixing process. Computer modeling can only calculate average properties at each node of the calculation domain: the mean velocity, mean concentration, and mean temperature.

Attempts have been made to assess the importance of vortex flow in the plasma torch on the properties of the plasma jet emanating from the 
torch [5], because most of the commercial plasma torches in use today employ vortex flows for stabilizing the arc within the torch. For this purpose we developed a two-dimensional model for a fully turbulent plasma jet with superimposed vortex flow, neglecting the previously mentioned large-scale flow structures. The model incorporated multiple time scales for velocity and temperature fluctuations and a density-weighted averaging for the density fluctuation effect. Results show that adding swirl to the flow field for confined and free jets induces strong axial and radial pressure gradients near the nozzle exit, causing a rapid decay of the axial velocity with increasing distance from the nozzle. Comparisons with cold flow show similar trends close to the nozzle exit, but further downstream, the axial velocities increase again, especially for larger swirl numbers. Comparisons of theoretical predictions based on the present model with available experimental data are shown in Fig. 2 for the centerline fields of axial velocity, argon mole fraction and plasma temperature [5]. The subscripts "com" and "exp" refer to computed and experimental data, respectively. A good agreement exists for the axial velocity distribution, especially for the region $x>30 \mathrm{~mm}$. For the argon mole fraction, some deviations between prediction and experimental data are observed; the experimental data are higher than the predictions in the vicinity of the nozzle exit, while the opposite conditions prevail farther downstream.

More severe deviations are observed in the case of the temrerature fields. In Fig. 2, $\mathrm{T}_{\mathrm{sp}}$ represents the data taken from spectroscopic measurements [6] which are widely used for determining plasma temperatures in the hot, luminous part of the plasma jet, while $T_{p r}$ denotes the data measured with an enthalpy probe [3] which is particularly useful for the lower-temperature regions. Substantial deviations exist between these 
measurements. For example at $\mathrm{x}=20 \mathrm{~mm}, \mathrm{~T}_{\mathrm{sp}}$ is around $10^{4} \mathrm{~K}$, while $\mathrm{T}_{\mathrm{pr}}$ shows a value slightly above $6000 \mathrm{~K}$.

This result is not surprising in light of the previous discussion. Spectrometric measurements refer to the hot plasma only whereas probe measurements incorporate both the hot plasma and the entrained cold air.

The predictions in the temperature diagram refer to the unweighted temperature $\left(\mathrm{T}_{\mathrm{un}}\right)$ and the mass-weighted (or density-weighted) temperature. Again, these results clearly indicate the importance of air entrainment into the plasma jet.

\section{INJECTION OF PARTICULATES INTO A THERMAL PLASMA}

Injection of particulate matter (solids or liquids) into a thermal plasma represents one of the approaches used in thermal plasma processing. The injected particles or droplets are usually treated as a dispersed phase, governed by the equation of motion and the rate equations for heat and mass transfer in Lagrangian coordinates. A stochastic approach has been introduced to take particle dispersion into account due to turbulent fluctuations by randomly sampling instantaneous flow fields [7]. Threedimensional effects were also considered which are mainly due to particle injection and the presence of a swirl component. A modified approach for investigating noncontinuum effects on piasma-particle heat transfer shows that the heat conduction jump approach overestimates the reduction of heat transfer for smaller particle or droplet sizes. Comparisons of theoretical predictions based on the present model with available experimental data are, in general, in reasonable agreement. 


\section{PLASMA REACTORS FOR PLASMA SYNTHESIS AND PLASMA CVD}

Injection of small mass particulates into a thermal plasma is not a trivial problem for two reasons: (1) The high viscosity of plasmas is detrimental for the penetration of particles into the plasma. Particles with insufficient inertia may "bounce off" the plasma. (2) In regimes of steep temperature gradients the thermophoresis effect may prevent particles from entering the hot plasma. In designing thermal plasma reactors these facts need to be considered in addition to making provisions for mixing and sufficient dwell time of the particles in the hot plasma. The latter is necessary to ensure complete evaporation of particulates for the ensuing gas phase reactions. Finally, the desired products have to be quenched to prevent possible undesirable reactions.

Experience with a gas-shrouded D.C. plasma reactor [8] demonstrated the necessity for more advarced designs. Fig. 3 shows a sketch of a triplecathode arc device with central injection into the temperature valley formed by the three converging arc columns [9]. This reactor may be considered as the forerunner for the Triple Torch Plasma Reactor (TTPR) which has been extensively used for plasma synthesis as well as for plasma CVD [10]. Fig. 4 shows a schematic of this reactor in this case designed for plasma CVD. The reactor contains three identical plasma torches. Each torch can operate in the range from 100 to 700 Amperes. Arc voltages are in the range from 28 to 53 Volts with pure argon or mixtures of argon and diatomic gases such as hydrogen. The torches are cooled by distilled water. The efficiency of the torches fall between $45-52 \%$ depending on the operating conditions.

These three torches are connected to a linkage which provides tilting as well as longitudinal motion. The linkage is driven by four stepping motors which are controlled by an IBM-PC. The angle between the torch and 
the center line can be varied from $14^{\circ}$ to $40^{\circ}$ with a neutral position at $30^{\circ}$ with respect to the center line. The torches can also move longitudinally along their own axes by about $10 \mathrm{~cm}$. The linkage system is mounted on a water cooled flange which sits on top of a vacuum chamber. The torches penetrate into the chamber through three flexible welded metal bellows, which offer the necessary flexibility for changing the orientation of the torches, ensuring at the same time vacuum tightness of tine chamber. The reactor vessel consists of a double wall, water cooled chamber, $50 \mathrm{~cm}$ in diameter and $64 \mathrm{~cm}$ in height. The stainless steel shell inside the chamber ensures corrosion resistance when exposed to chemical reactants during operation. A number of flanges provide access for diagnostics and feedthroughs.

Over the past years a novel Counter-Flow Liquid Injection Plasma Synthesis (CF LIPS) reactor has been developed to produce ceramic powders. By using a counter flow plasma configuration, entrainment of reactant particles into the plasma is improved compared to conventional injection methods. The counter flow process also creates large recirculation zones which increase the residence time to more than $100 \mathrm{~ms}$ as predicted by modeling results which will be discussed later on. The long residence time ensures complete evaporation and decomposition of precursor particles and complete reactions to the desirable products. Also, the process employs liquid precursors rather than solids, resulting in less contamination of products from unevaporated reactants and, at the same time, it eliminates problems of continuous and uniform feeding of fine particles encountered with previous reactors. Fig. 5 shows a schematic of this reactor. The main components of the reactor are: a dc plasma torch, a high pressure water-cooled 
atomizer/injection probe, a dcuble-wall water-cooled reaction chamber and a water-cooled collection chamber.

Precursor solutions have been prepared by mixing metal nitrates in their stoichiometric ratios and these solutions have been fed through a watercooled pneumatic atomizer where the solution is atomized into fine droplets by a a narrow jet of atomizing gas of either argon or oxygen or compressed air. The fine droplets are than carried by the atomizing gas and are sprayed counter-flow into the plasma jet. An xyz translator has been used to align the probe with the plasma jet so that the probe tip has been enveloped by the plasma completely and symmetrically (as illustrated in Fig. 5). By doing so, reactants have been heated effectively for complete reactions. Powders have been collected in the collection chamber and in a filter at the exhaust, but most of the powders have been deposited in the inner wall of the reaction chamber. The previously mentioned long dwell time of particulates in this reactor is a consequence of recirculating flows. The flow field calculations [11] based on laminar flow show two major recirculating regions (Fig. 6). In addition, the temperature within these recirculation zones remains relatively uniform as shown in Fig. 6.

Both the TTPR and the CF LIPS reactor have been extensively used for the experiments which will be discussed in the following sections.

\section{SYNTHESIS OF FINE POWDERS}

Before the new plasma reactors became available for experimentation, a simple convection-stabilized arc arrangement was used for synthesizing $\mathrm{Si}_{3} \mathrm{~N}_{4}$ [8]. Submicron $\alpha-, \beta-$, and amorphous-phase $\mathrm{Si}_{3} \mathrm{~N}_{4}$ particles were synthesized using metallic silicon and ammonia as reactants. Injection of ammonia at different locations of the reactor resulted in different yields. A 
maximum yield of $85 \mathrm{wt} \%$ has been achieved by injecting $\mathrm{NH}_{3}$ at both downstream and upstream locations of the reactor. The powders synthesized in this way contained approximately $60 \mathrm{wt} \%$ silicon nitride in crystal form with equal amounts of $\alpha$ and $\beta$ phases. The remainder consisted of the amorphous phase. The average size of the particles ranged from 50 to $90 \mathrm{~nm}$, with a standard deviation of 1.47-1.87 depending on the location of ammonia injection. Seeding with 1 and $10 w t \%$ of preexisting silicon nitride particles for fostering heterogeneous nucleation did not improve the yield, but it changed the particle size distribution.

Characterization of the produced $\mathrm{Si}_{3} \mathrm{~N}_{4}$ particles which were formed by gas-condensed phase reactions revealed that the morphologies consisted of $\beta$ $\mathrm{Si}_{3} \mathrm{~N}_{4}$ prisms, $\alpha-\mathrm{Si}_{3} \mathrm{~N}_{4}$ matte, $\alpha-\mathrm{Si}_{3} \mathrm{~N}_{4}$ needles, and spagheti-like whiskers. In contrast, for the homogeneously nucleated particles in the gas phase, the morphologies included dendrites, needles, platelets, and amorphous particles. Most of the particles formed were aggregates with particle size distributions ranging from 500 to $1500 \AA$ depending on the location of injection of the reactants.

Ceramic powders of carbides, aluminum nitride, oxides, solid solutions, magnetic and non-magnetic spinels, superconductors, and composites have been successfully synthesized in a Triple DC Torch Plasma Jet Reactor (TTPR) and in a single DC Plasma Jet Reactor. All the ceramic powders with the exception of AIN were synthesized using a novel liquid injection method developed to overcome the problems associated with solid injection, in particular for the single DC plasma jet reactor, and to realize the benefits of gas phase reactions.

In the following the results of the various experiments will be briefly summarized. 
Aluminum nitride powder has been produced by using the TTPR system operating in the non-transferred arc mode $[10,12]$ using an argon/nitrogen mixture as plasma gas. Fine aluminum powder with a medium size of 1 to $2 \mu \mathrm{m}$ was used as a precursor. The Al powder was injected with a water cooled feeding tube into the temperature valley between the converging plasma jets using nitrogen as carrier gas. The product collected from a quartz tube downstream of the plasma jets consisted of the hexagonal and the cubic AlN phase with very little unreacted $\mathrm{Al}$. The powder size distribution obtained by centrifugal sedimentation revealed a peak around $0.3 \mu \mathrm{m}$.

Zirconium carbide has been synthesized using $(\mathrm{RO})_{\mathbf{4}} \mathrm{Zr}$, a liquir organometallic precursor in the TTPR. Thermodynamic equilibrium calculations indicate that in the temperature range from 1,800 to $3,800 \mathrm{~K}$, zirconium carbide can be formed by plasma pyrolysis. These calculations also suggest that by adding some $\mathrm{CO}_{2}$ to the plasma gas, the excess carbon derived from the precursor can be removed as shown in Fig. 7. The carbide content reaches $100 \%$ when 11 moles of $\mathrm{CO}_{2}$ are added to each nole of butoxide. Below this critical point, excess carbon shows up, and above this point, the carbide changes into zirconia. The sharp peak is an indication that fine tuning of the experimental conditions is crucial for obtaining a pure product.

In parallel to the TTRP experiments, plasma synthesis has been performed in a previously described single torch plasma jet reactor using counter-flow liquid injection. In this counter-flow liquid injection plasma reactor, the temperature field is rather uniform and extends much further downstream in the reaction chamber. Large recirculation zones developed above and below the stagnation region of the two flows. This recirculation increases the residence time and mixing of the reactants in the plasma. Modeling results indicate that the residence time of materials in a $25 \mathrm{~cm}$ long 
reaction chamber is more than $120 \mathrm{~ms}$ which is two orders of magnitude higher than the conventional parallel injection. Figure 6 shows the modeling results of temperature and flow fields. A large variety of ceramic powders has been synthesized by this method. The powders produced are uniform, single phased, spherical and non-agglomerating particles.

Consistently, more than $70 \%$ of the powders by volume is within $1-2 \mu \mathrm{m}$. The results are highly reproducible and agree well with our modeling predictions. Table 1 is a summary of the materials produced by this CF-LIPS.

Table 1. SUMMARY OF DC PLASMA COUNTER-FLOW LIPS

CARBIDES

OXIDES

SOLID SOLUTIONS

SPINELS

SUPERCONDUCTORS

COMPOSITES
$\mathrm{B}_{4} \mathrm{C}, \mathrm{SiC}$ and $\mathrm{ZrC}$

$\mathrm{Al}_{2} \mathrm{O}_{3}, \mathrm{MgO}, \mathrm{NiO}, \mathrm{CoO}, \mathrm{ZrO}_{2}, \mathrm{Y}_{2} \mathrm{O}_{3}$, and $\mathrm{CeO}_{2}$

$\mathrm{Y}_{2} \mathrm{O}_{3}$, and $\mathrm{CeO}_{2}: \mathrm{Y}_{2} \mathrm{O}_{3}$, stabilized $\mathrm{ZrO}_{2}$

$\mathrm{Co}, \mathrm{Mg}, \mathrm{Ni}$, and $\mathrm{Zn}$ aluminates

$\mathrm{Co}, \mathrm{Mg}, \mathrm{Ni}, \mathrm{Cu}$, and $\mathrm{Zn}$ ferrites

$\mathrm{Co}, \mathrm{Mg}, \mathrm{Ni}$, and $\mathrm{Cu}$ chromites

$\mathrm{YBa}_{2} \mathrm{Cu}_{3} \mathrm{O}_{7-\mathrm{x}}, \mathrm{Bi}-\mathrm{Sr}-\mathrm{CA}-\mathrm{Cu}-\mathrm{O}_{\mathbf{x}}$

(2212 and 2223 phases)

$[\mathrm{ZrCl}]_{\mathrm{x}}:\left[\mathrm{ZrO}_{2}\right]_{\mathrm{y}}$

The most recent experiments considered the production of spinels [13] and the results revealed spherical shaped, smooth particles in a size range from submicron to micron as shown in Fig. 8. By varying the operating parameters, in particular the precursor concentration, the particle size can be controlled. Relationships between particle size and operating parameters will be further investigated. 
Crystallographic stuci $s$ indicated that the as-produced spinels shown in Fig. 8 were slightly less dense than ideal spinels and their stoichiometries also depart from the ideal situation. Electron microprobe analysis revealed some minor phases besides the spinel phase.

\section{PLASMA CHEMICAL VAPOR DEPOSITION}

Initial experiments have been performed for the deposition of diamond coatings on Si wafers using the TTPR with methane as the carbon source [14]. The wafer surface was treated with diamond paste or beta SiC seed particles. The methane concentration varied from $3.5 \%$ to $6.5 \%$ with the balance being hydrogen. Well faceted diamond crystallites were deposited on the surface of the wafers, forming a continuous one particle thick coating. Nearly all crystallites showed $\{111\}$ growth planes with $\{$ h00\} growth planes also observed. The formation of diamond has been confirmed with X-ray diffraction and Raman spectroscopy. At higher methane content (6.5\%), crystallite sizes decreased and $\langle$ h00 $>$ growth became more prominent. To our knowledge, well faceted diamond crystals formed at such a high methane content at one atmosphere, have not been observed before. Deposition rates were typically in the range from 40 to $60 \mu \mathrm{m}$ per hour.

Transmission electron microscopy (TEM) has been used for characterization of diamond films produced in the TTPR [15]. The results have shown that an intermediate $\mathrm{SiC}$ phase and amorphous $\mathrm{Si}$ exist between the single crystal silicon substrate and the diamond films. The size, orientation, and distribution of $\beta$-SiC have been characterized by dark-field imaging techniques. The result revealed a predominant $[100]_{\mathrm{SiC}} \mathrm{I}^{\prime}[100]_{\mathrm{Si}}$ distribution of $\mathrm{SiC}$. 
The selected area channeling pattern (SACP) technique has allowed us to determine that crystallites grown with their (100) directions normal to the substrate have ferver defects than those grown with (111) normals.

Further studies of diamond deposition during the past year using both the TTPR and the CF LIPS reactor $[16,17]$ may be summarized as follows: (1) Diamonds can be deposited on both carbide-forming materials such as silicon, molybdenum, tungsten, and tantalum and non-carbide forming materials such as copper, nickel, and tantalum. Diamond has also been deposited on stainless steel.

(2) It has been found that the methane concentration has a pronounced effect on the siz: of the constituent diamond crystals in the film. The higher the concentration, the smaller the grain size (Fig. 9a-c). This observation may be interpreted by the increased number of secondary nucleations sites formed at increased methane concentration. When the methane concentration exceeds a certain threshold, crystallinity vanishes and only cauliflower-type balls are produced (Fig. 9 d).

(3) In thermal plasmas diamond can be produced with different organic liquid precursors, including (a) alcohols, (b) ketones, (c) halogenated compounds, and (d) aromatic compounds. The high energy content of thermal plasmas enables the pyrolysis of various precursors. The growth rate of diamond films achieved with ethanol may be as high as $1,000 \mu \mathrm{m} /$ hour (Fig. 10).

(4) It has been found that the substrate temperature affects the morphology and the direction of growth of crystals and films. At low temperatures $\left(<1,000^{\circ} \mathrm{C}\right)$, the growth surface is dominated by the (111) planes while at higher temperatures $\left(>1000^{\circ} \mathrm{C}\right)$ by the $(100)$ planes. 
(5) The process pressure has been found to affect the growth rate of the diamond films. The optimum pressure is around 270 Torr at a substrate temperature of $1,000^{\circ} \mathrm{C}$. Above or below this pressure, the deposition rates are lower.

(6) The requirement for substrate pre-treatment is material dependent under thermal plasma conditions. Lapping with diamond paste greatly increases the initial nucleation density on silicon wafers but this seems to have little effect on metal substrates.

\section{REFERENCES}

1. R. Spores and E. Pfender, "Flow Structure of a Turbulent Thermal Plasma Jet," Surface and Coatings Technology, 37, 251(1989). The paper was also presented at the National Thermal Spray Conference, Cincinnati, OH, Oct. 1988.

2. E. Pfender, J. Fincke, and R. Spores "Entrainment of Cold Gas into Thermal Plasma Jets," Plasma Chem. and Plasma Proc., Vol. 11(4), 529 (1991).

3. M. Brossa, and E. Pfender "Probe Measurements in Thermal Plasma Jets", Plasma Chemistry and Plasma Processing, Vol. 8, No. 1, 75 (1988).

4. A. Capetti and E. Pfender, "Probe Measurements in Argon Plasma Jets Operated in Ambient Argon,"Plasma Chem. and Plasma Proc., Vol. 9, No. 2, 329 (1989).

5. Y.P. Chyou and E. Pfender, "Modeling of Plasma Jets with Superimposed Vortex Flow,"Plasma Chem. and Plasma Proc., Vol. 9, No. 2, 291 (1989).

6. E. Fleck, "Temperature and Velocity Measurements of Thermal Plasma Jets," Ph.D. Thesis, Department of Mechanical Engineering, University of Minnesota, Minneapolis (1986).

7. Y.P. Chyou and E. Pfender, "Behavior of Particulates in Thermal Plasma Flows,"Plasma Chem. and Plasma Proc., Vol. 9, No. 1, 45 (1989). 
8. Yl Chang, R.M. Young, and E. Pfender, "Silicon Nitride Synthesis in an Atmospheric Pressure Convection-Stabilized Arc," Plasma Chem. and Plasma Proc., Vol. 9, No. 2 , 277 (1989).

9. R.M. Young and E. Pfender, "A Novel Approach for Introducing Particulate Matter into Thermal Plasmas: The Triple-Cathode Arc," Plasma Chem. and Plasma Proc., Vol. 9 (4), 465 (1989).

10. Z.P. Lu, and E. Pfender "Synthesis of AIN Powder in a Triple Torch Plasma Reactor," Proc. of the 9th International Symposium on Plasma Chemistry, Pugnochiuso, Italy, Vol. 2, 675 (1989).

11. Seungho Paik, Xi Chen, P. Kong and E. Pfender, "Modeling of a Counter-flow Plasma Reactor," Plasma Chem. and Plasma Proc.,Vol. 11 (2), 229(1991).

12. Z.P. Lu and E. Pfender, "DC Plasma Synthesis of Aluminum Nitride Ceramic Powders," in Materials Research Society Symposium Proceedings, Vol. 180, Better Ceramics Through Chemistry IV, Materials Research Society, Pittsburgh, PA, p. 857 (1990).

13. T.W. Or, P.C. Kong, and E. Pfender, "Counter-Flow Liquid Injection Plasma Synthesis of Spinel Powders," Plasma Chem. and Plasma Proc., Vol. 12, No.2 (to be published in June 1992).

14. Z.P. Lu, L. Stachowicz, P. Kong, E. Pfender, and J. Heberlein, "Diamond Synthesis by DC Thermal Plasma CVD at 1 atm," Plasma Chem. and Plasma Proc. Vol. 11 (3), 387(1991).

15. C. Tsai, W. Gerberich, Z.P. Lu, J. Heberlein, and E. Pfender, "Characterization of thermal plasma CVD diamond coatings and the intermediate SiC phase," J. Mater. Res., Vol. 6 (10), 2127 (1991).

16. Z.P. Lu, L. Stachowicz, E. Pfender and J. Heberlein, "Effects of Process Parameters on Chemical Vapor Deposition of Diamond in Thermal Plasmas " Proc. of the 2nd. International Symposium on Diamond Materiais," Vol. 91-8, The Electrochemical Society, Pennington, New Jersey, pp. 107-114, 1991.

17. Q.Y. Han, T.W. Or, Z.P. Lu, J. Heberlein and E. Pfender, "High Rate Deposition of Diamond Using Liquid Organic Precursors in Atmospheric Plasmas," Proc. of the 2nd. International Symposium on Diamond Materials," Vol. 91-8, The Electrochemical Society, Pennington, New Jersey, pp. 115-122, 1991. 


\section{PUBLICATIONS}

. . (papers published during the reporting period )

\section{a) Joxinal Publications}

1. R. Spores and E. Pfender, "Flow Structure of a Turbulent Thermal Plasma Jet," Surface and Coatings Technology, Vol. 37, 251 (1989).

2. Y.P. Chyou and E. Pfender, "Behavior of Particulates in Thermal Plasma Flows," Plasma Chem. and Plasma Proc., Vol. 9 (1), 45 (1989).

3. Yl Chang, P. Kong and E. Pfender, "Characterization of Silicon Nitride Particles Synthesized in an Atmospheric-Pressure Convection-Stabilized Arc," Plasma Chem. and Plasma Proc., Vol. 9 (1), 73 (1989).

4. Yl Chang, R.M. Young and E. Pfender, "Silicon Nitride Synthesis in an Atmospheric Pressure Convection-Stabilized Arc," Plasma Chem. and Plasma Proc. Vol. 9 (2), 277 (1989).

5. Y.P. Chyou and E. Pfender, "Modeling of Plasma Jets with Superimposed Vortex Flow," Plasma Chem. and Plasma Proc., Vol. 9 (2), 291 (1989).

6. A. Capetti and E. Pfender, "Probe Measurements in Argon Plasma Jets Operated in Ambient Argon," Plasma Chein. and Plasma Proc., Vol. 9(2), 329 (1989).

7. R.M. Young and E. Pfender, "A Novel Approach for Introducing Particulate matter into Thermal Plasmas; The Triple-Cathode Arc," Plasna Chem. and Plasma Proc., Vol. 9 (4), 465 (1989).

8. E. Pfender, J. Fincke, and R. Spores "Entrainment of Cold Gas into Thermal Plasma Jets," Plasma Chem. and Plasma Proc., Vol. 11(4), 529 (1991).

9. Z.P. Lu, L. Stachowicz, P. Kong, J. Heberlein and E. Pfender, "Diamond Synthesis by D.C. Thermal Plasma CVD at One Atmosphere," Plasma Chem. and Plasma Proc., Vol. 11 (3), 387 (1991).

10. S.H. Paik, Xi Chen, P. Kong and E. Pfender, "Modeling of a Counter-Flow Plasma Reactor," Plasma Chem. and Plasma Proc., Vol 11 (2), 229 (1991).

11. C. Tsai, W. Gerberich, Z.P. Lu, J. Heberlein and E. Pfender, "Characterization of Thermal Plasma CVD Diamond Coatings and the Intermediate SiC Phase," Journal of Materials Research, Vol. 6 (10), 2127 (1991). 
12. T.W. Or, P.C. Kong, and E. Pfender, "Counter-Flow Liquid Injection Plasma Synthesis of Spinel Powders," Plasma Chem. and Plasma Proc., Vol. 12 (2), 189 (1992).

13. Z.P. Lu, J. Heberlein, and E. Pfender, " Process Study of Thermal Plasma Chemical Vapor Deposition of Diamond, Part I: Substrate Material, Temperature, and Methane Concentration," Plasma Chem. and Plasma Proc., Vol. 12 (1), 35 (1992).

14. Z.P. Lu, J. Heberlein, and E. Pfender, " Process Study of Thermal Plasma Chemical Vapor Deposition of Diamond, Part II: Pressure Dependence and Effect of Substrate Pre-treatment," Plasma Chem. and Plasma Proc., Vol. 12 (1), 55 (1992).

b) Other (Proceedings)

1. Z.P. Lu and E. Pfender, "Synthesis of AlN Powder in a Triple Torch Plasma Reactor," Proc. of the 9th Int. Symp. on Plasma Chem., Pugnochiuso, Italy, Vol. 2, 675 (1989).

2. P.C. Kong, and E. Pfender, "Plasma Synthesis of Fine Powders by Counter-Flow Liquid Injection," invited lecture, published in Combustion and Plasma Synthesis of High Temperature Materials, (peer reviewed) edited by Z.A. Munir and J.B. Holt, VCH Publishers, 1990.

3. Z.P. Lu, T.W. Or, L. Stachowicz, P. Kong and E. Pfender, "Synthesis of Zirconium Carbide in a Triple Torch Plasma Reactor Using Liquid Organometallic Zirconium Precursors," presented at the 1990 MRS Spring Meeting, accepted for publication in the Proceedings, (peer reviewed).

4. P. Kong, T.W. Or, L. Stachowicz and E. Pfender, "Plasma Synthesis of Fine Ceramic Powders by A Novel Counter-Flow Liquid Injection Method," Presented at the 1990 MRS Spring Meeting, Accepted for publication in the Proceedings, (peer reviewed).

5. T.W. Or, Z.P. Lu, L. Stachowicz, P. Kong and E. Pfender, "Counter-Flow Liquid Injection Plasma Synthesis (CF-LIPS) of Spinel Fine Powders," presented at the 1990 MRS Spring Meeting, accepted for publication in the Proceedings, (peer reviewed).

6. P.C. Kong and E. Pfender, "Thermal Plasma Synthesis of Fine Powders A Review," invited chapter to a monograph in Materials Processing Theory and Practices, ed. Prof. F.F.Y. Wang (1991). 
. 7. Z.P. Lu, T.W. Or, L. Stachowicz, J. Heberlein and E. Pfender, "Diamond CVD in Thermal Plasmas," Heat Transfer in Thermal Plasma Processing, Proceedings of the 27th National Heat Transfer Conf., ASME-HTD-Vol. 161, Minneapolis, MN, USA, July 1991, Ed. K. Etemadi and J. Mostaghimi, Americait Society of Mech. Engineers, New York, pp. 21 (1991). 


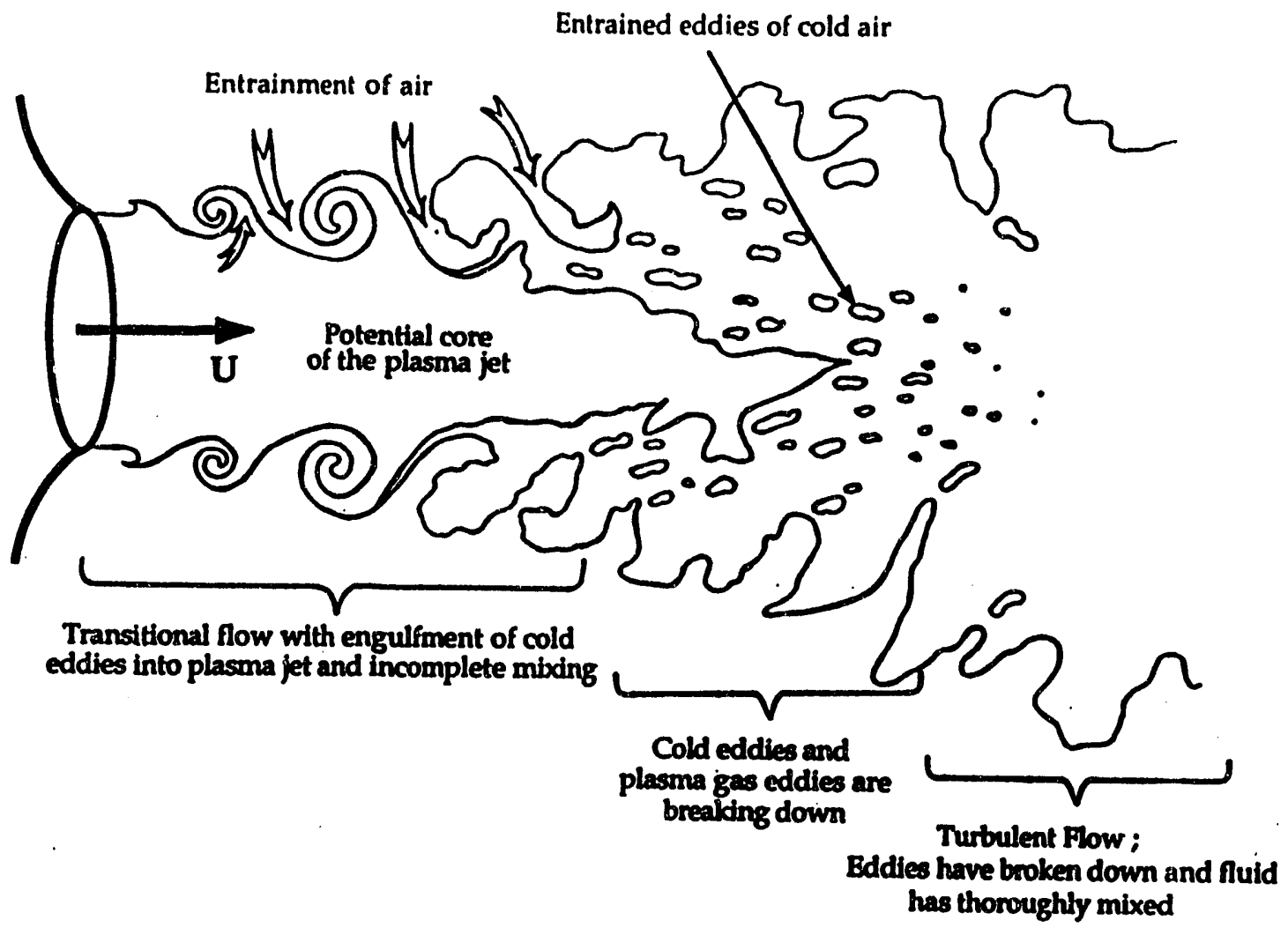

Fig. 1: Schematic of the main regions of a transitional plasma jet 

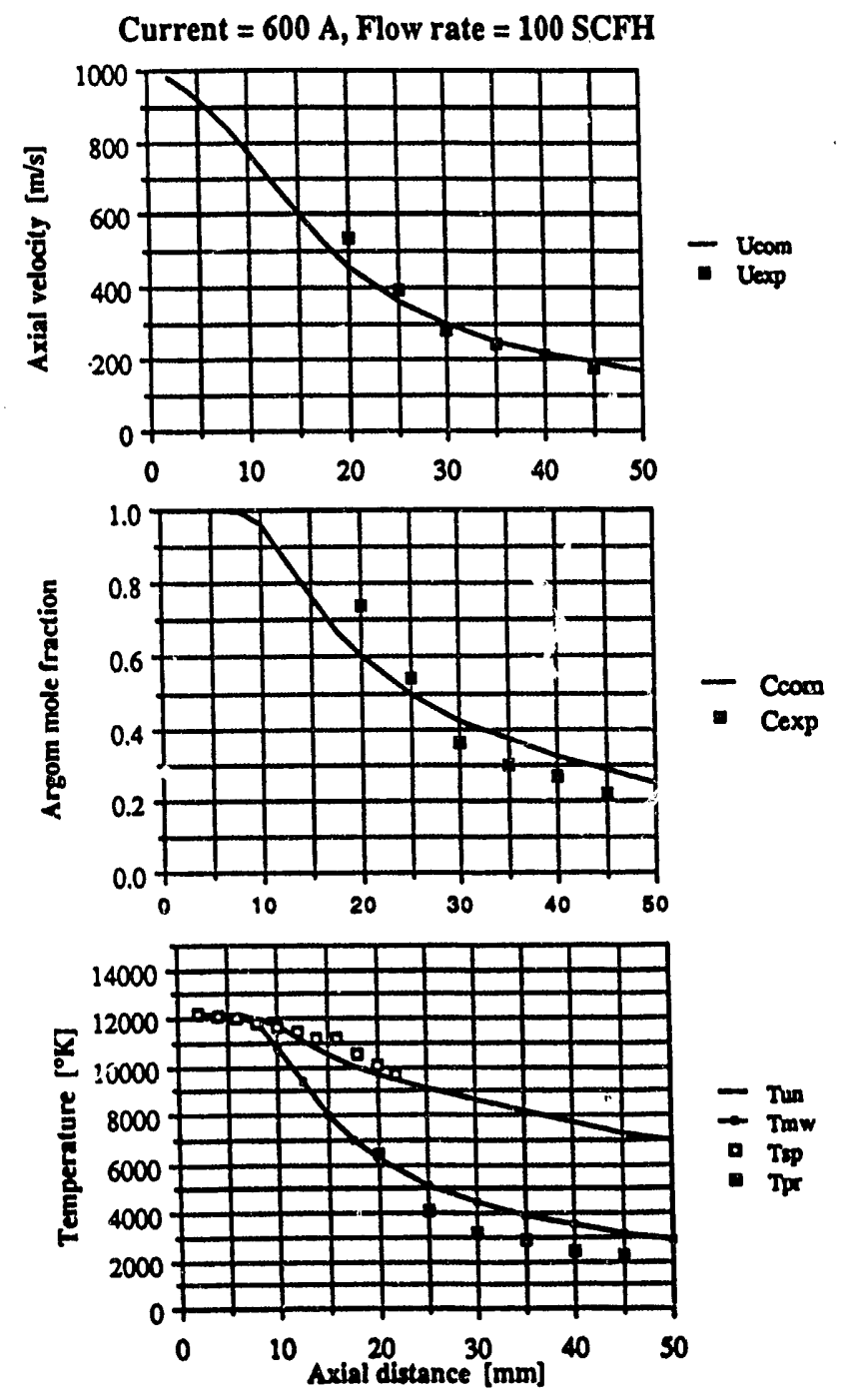

Fig. 2: Comparison of calculated centerline fields with experimental data 


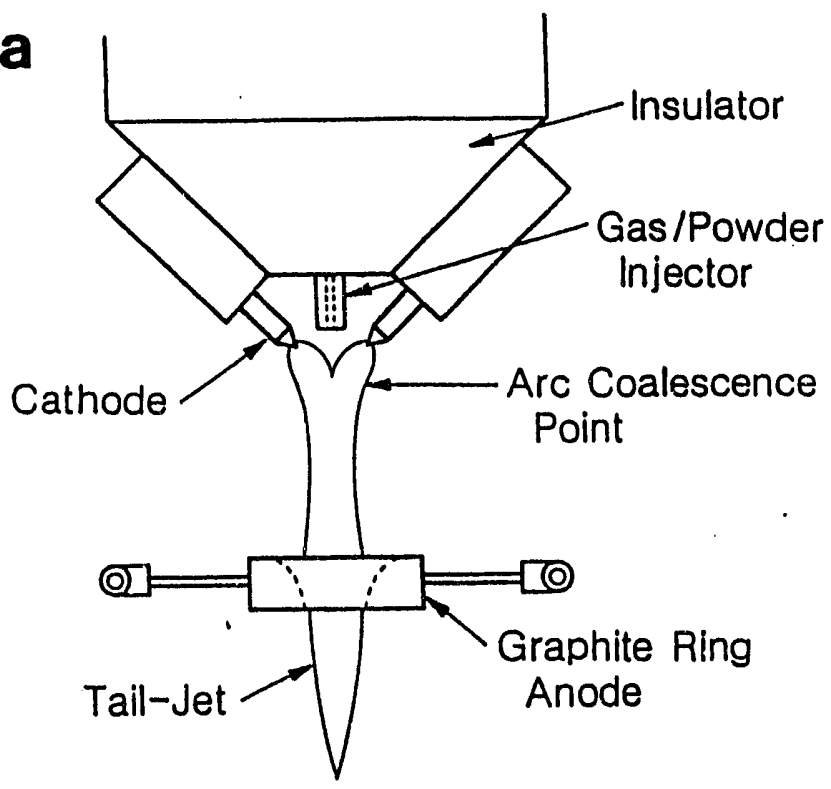

Fig. 3: Schematic of a triple-cathode arrangement 


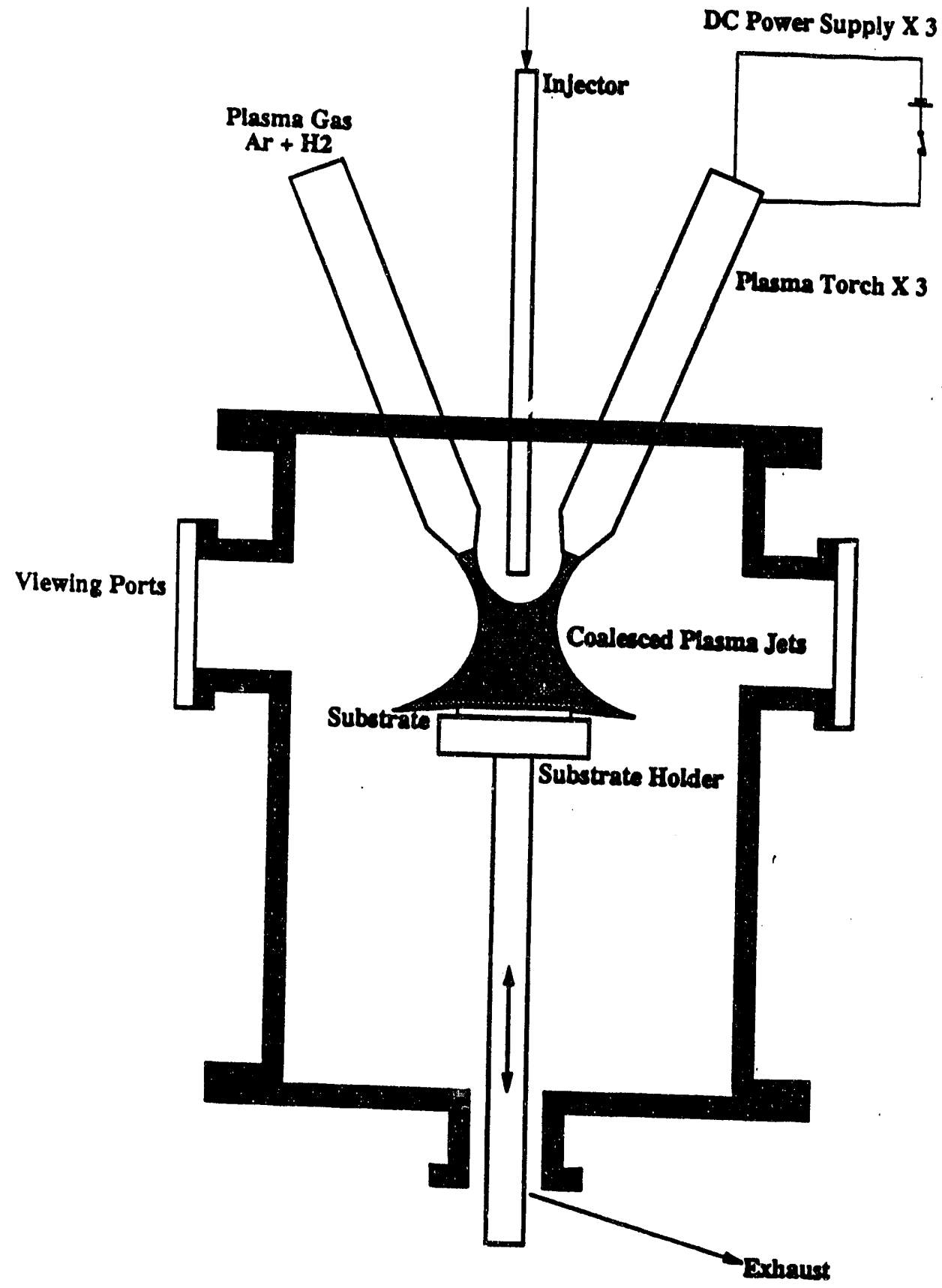

Fig. 4: Schematic of the triple-torch plasma reactor (TTPR) 


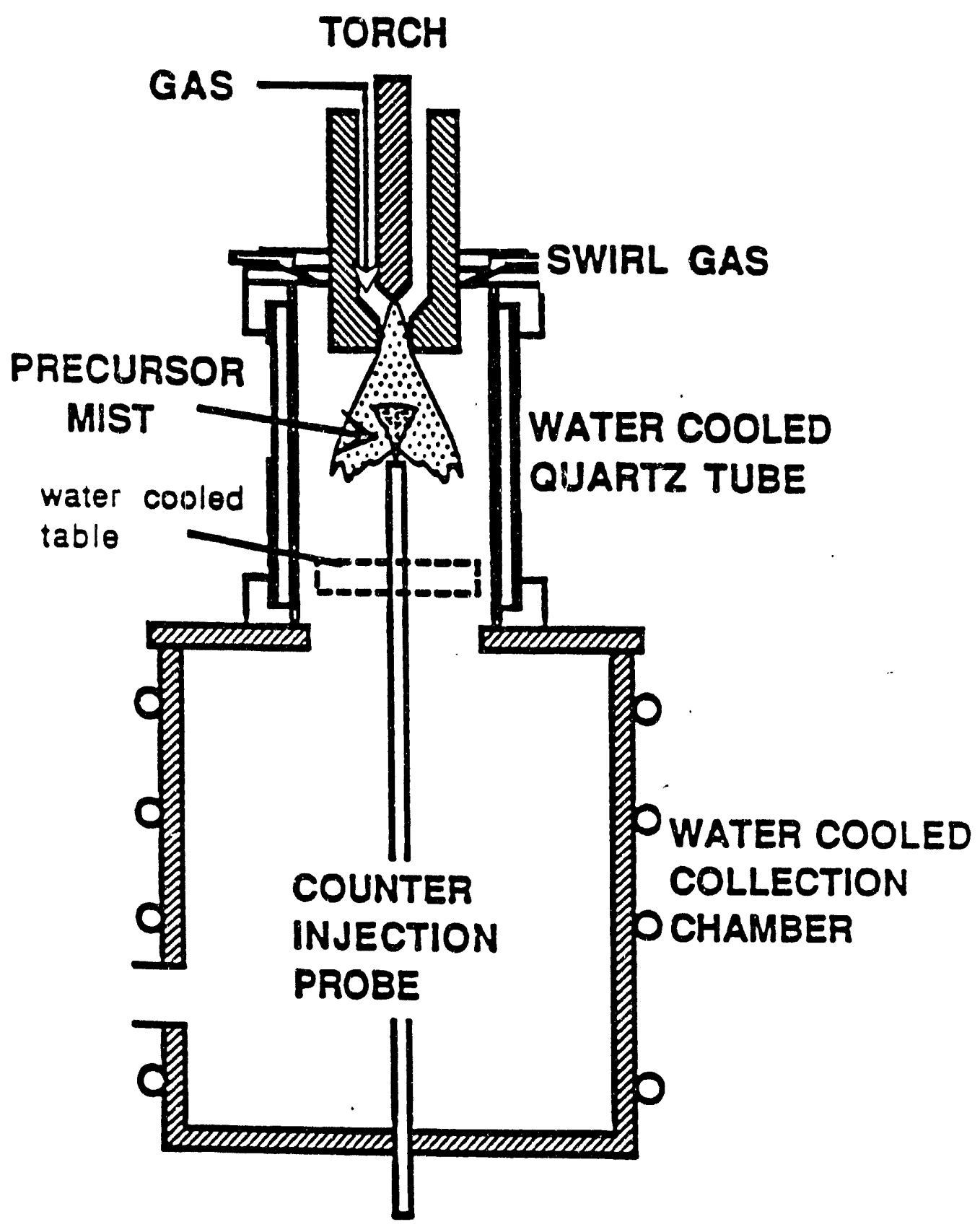

Fig. 5: Schematic of the CF LIPS reactor 


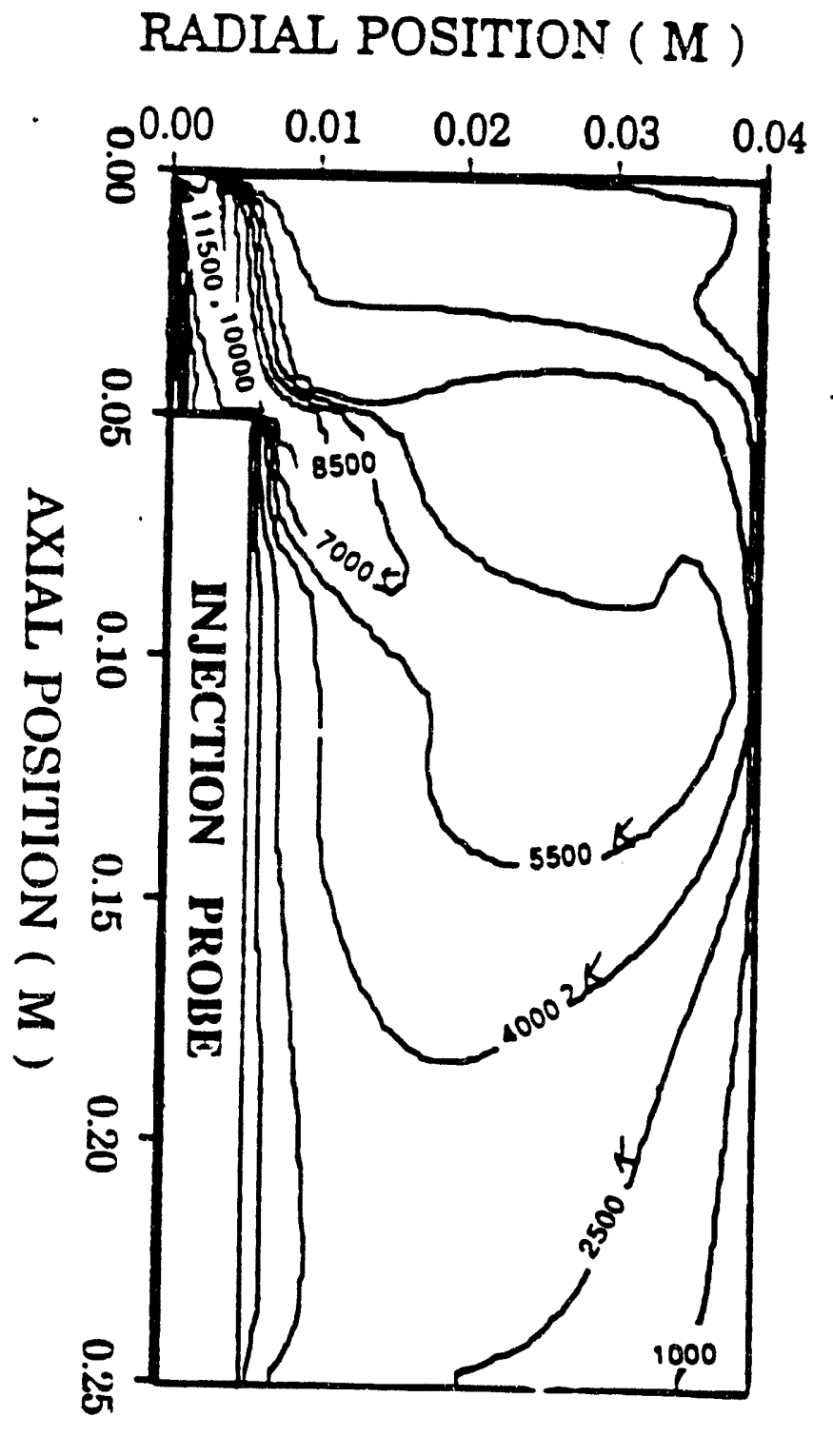

TEMPERATURE FIELD

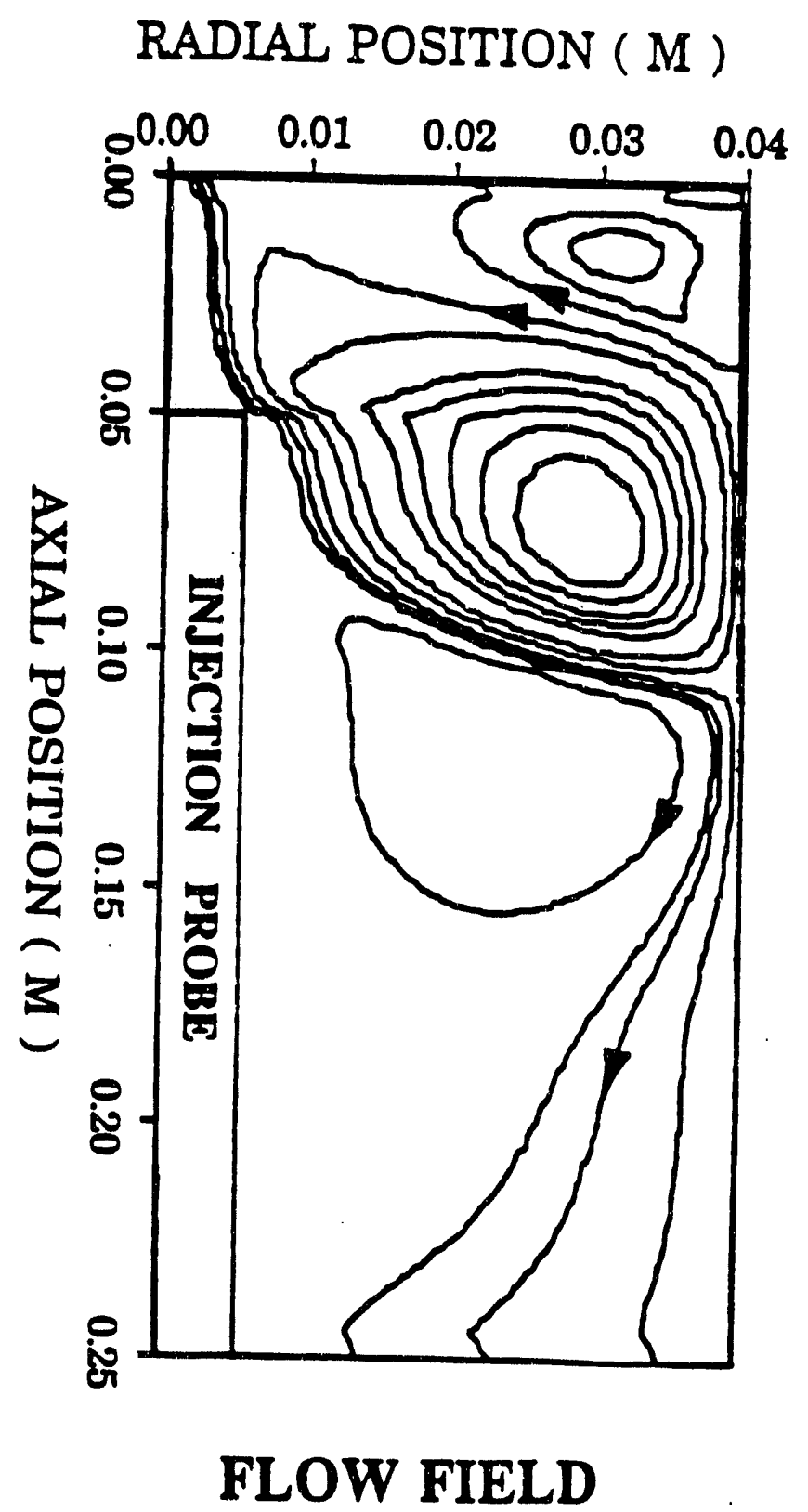

Fig. 6: Modeling results for the CF LIPS process 


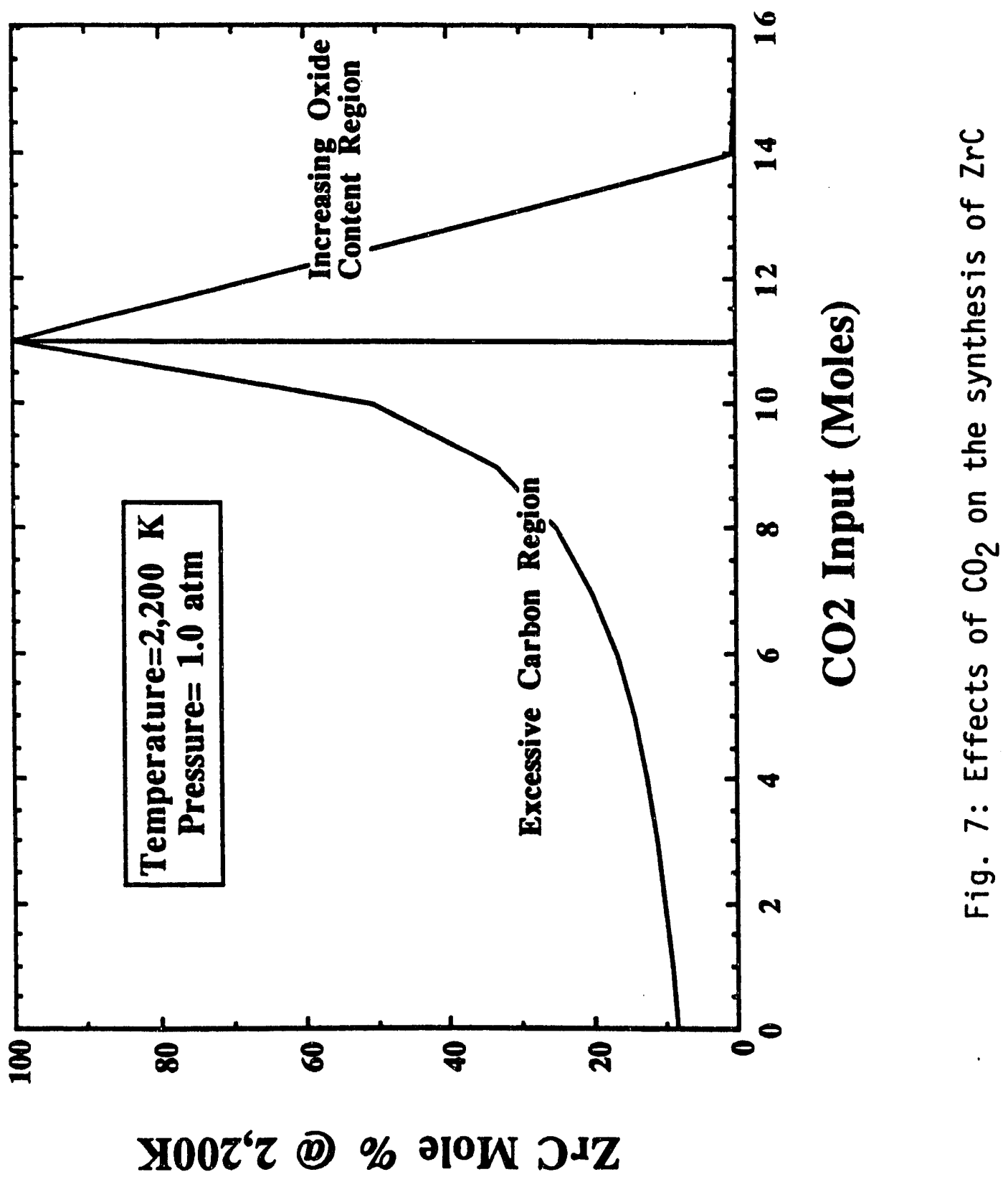


(a)

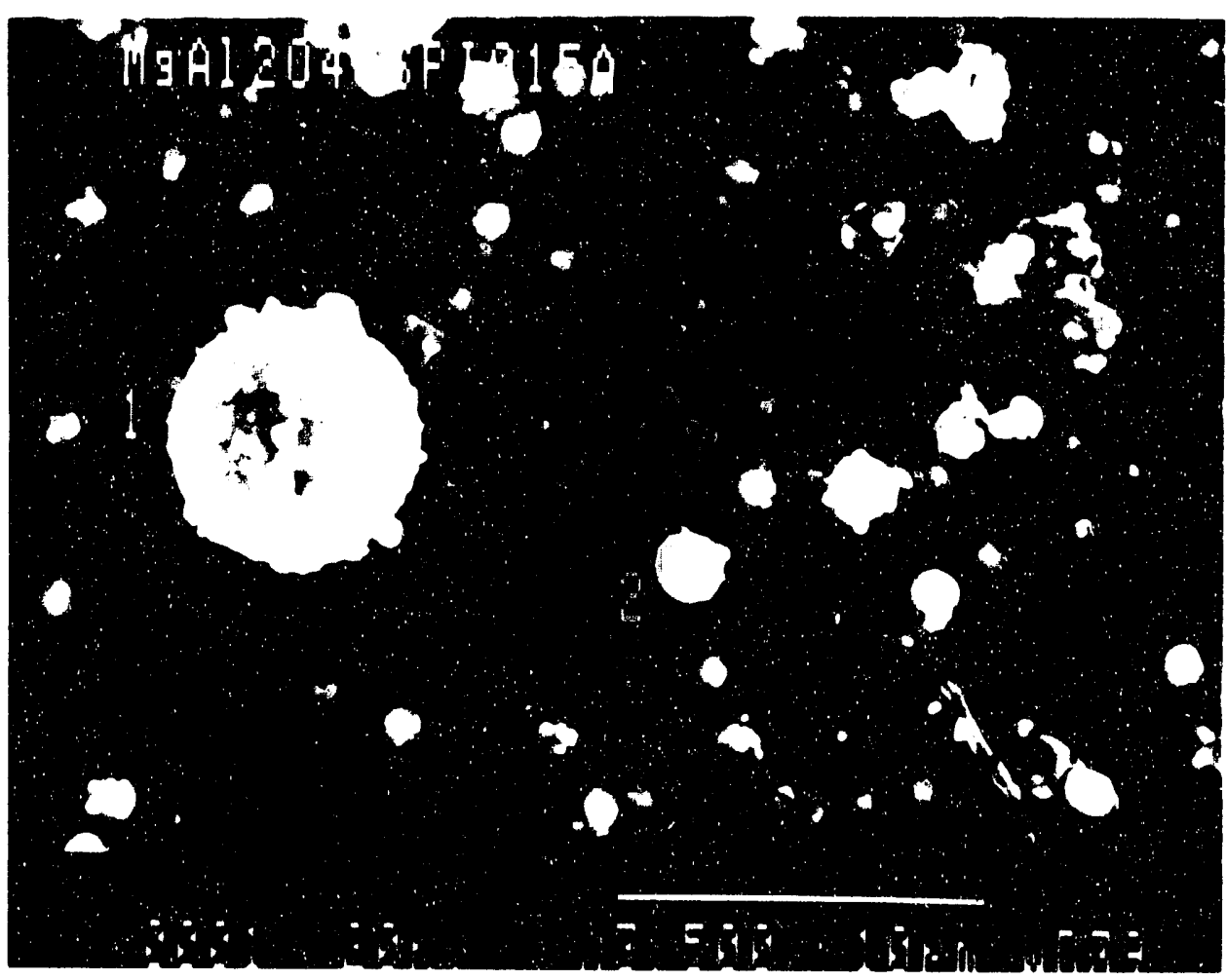

(b)

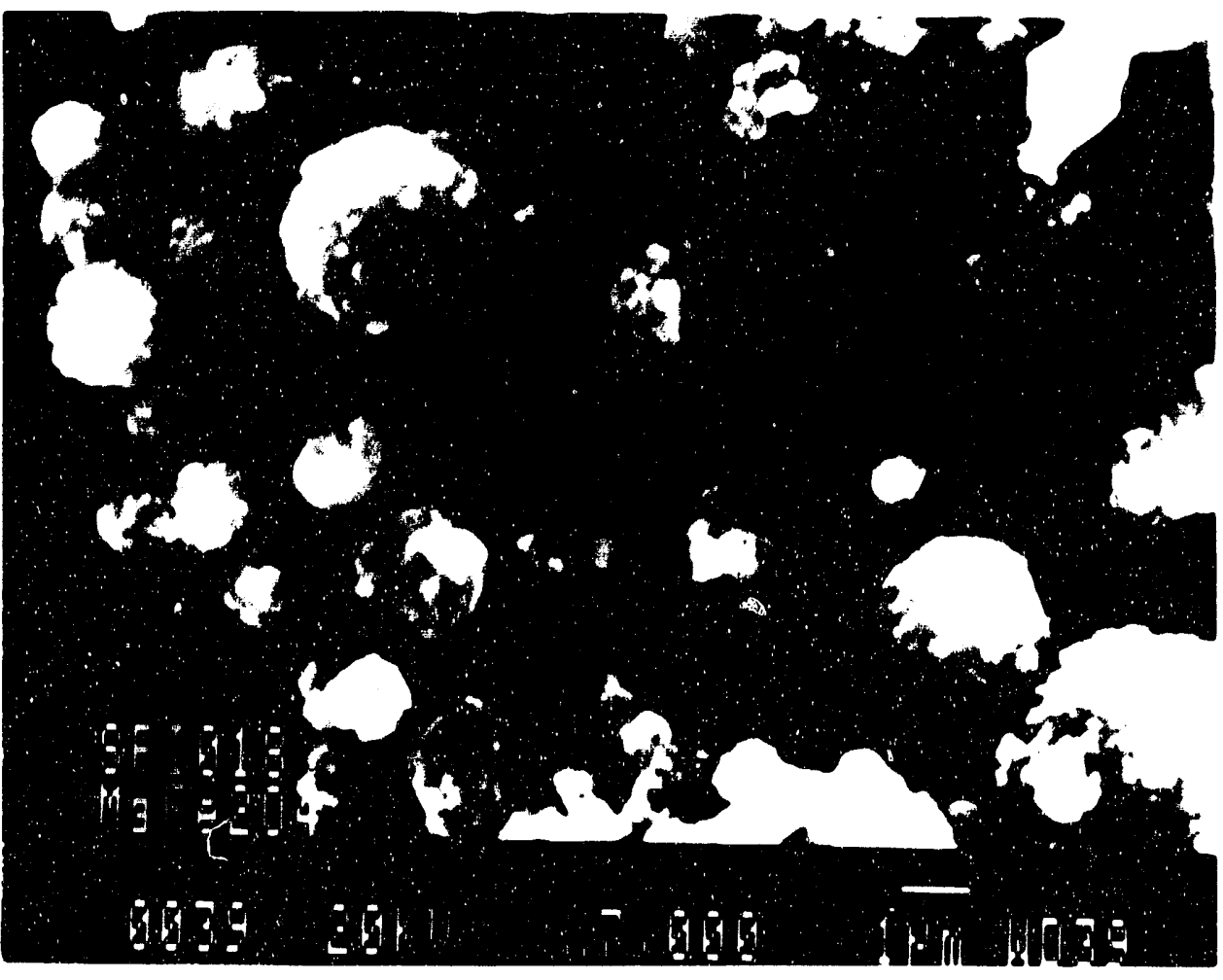

Fig. 8: SEM micrographs of spinel powders
(a) $\mathrm{MgAl}_{2} \mathrm{O}_{4}$
(b) $\mathrm{MgFe}_{2} \mathrm{O}_{4}$ 

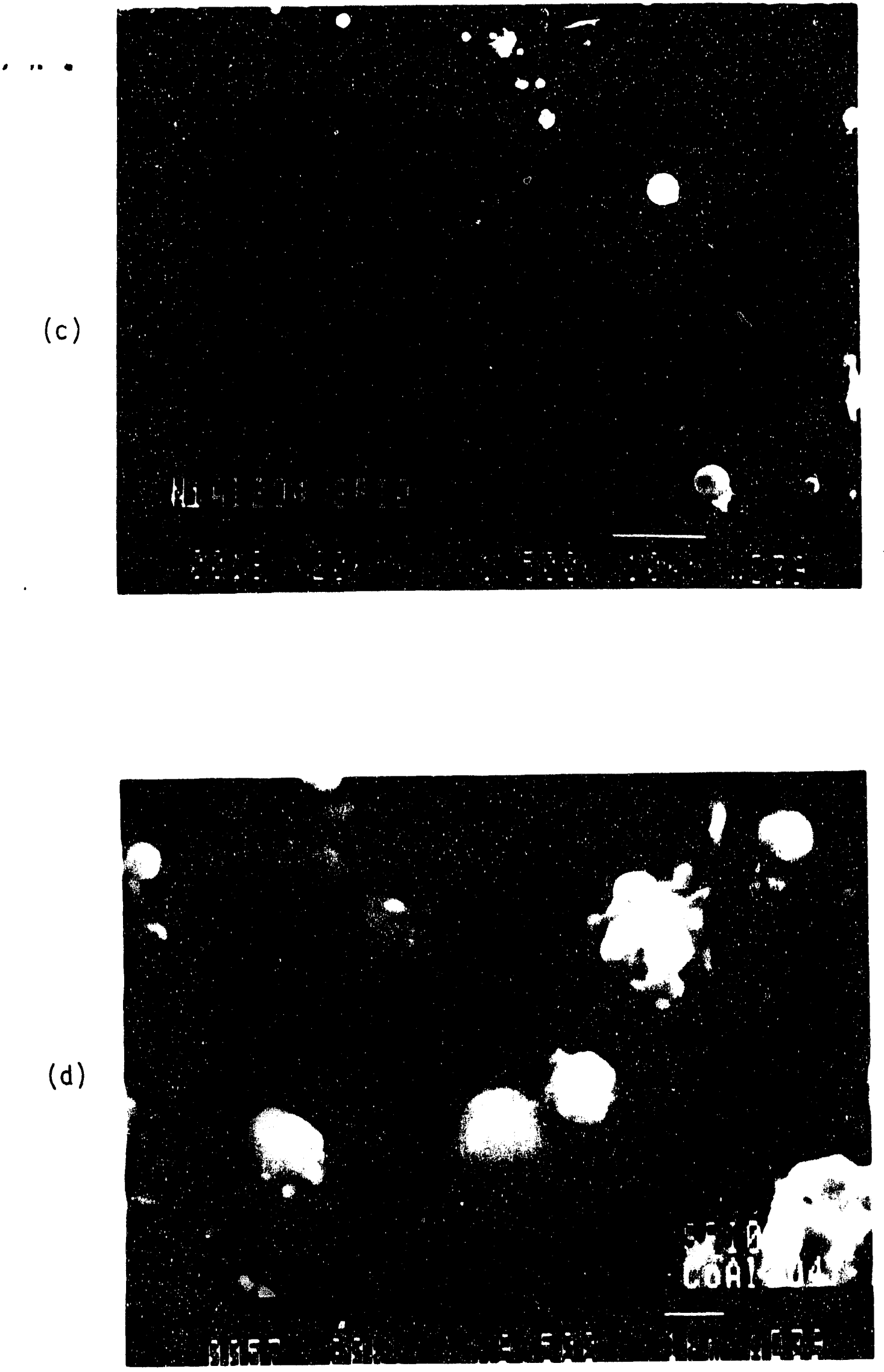

Fig. 8: SEM micrographs of spinel powders
(c) $\mathrm{NiAl}_{2} \mathrm{O}_{4}$
(d) $\mathrm{CoAl}_{2} \mathrm{O}_{4}$ 


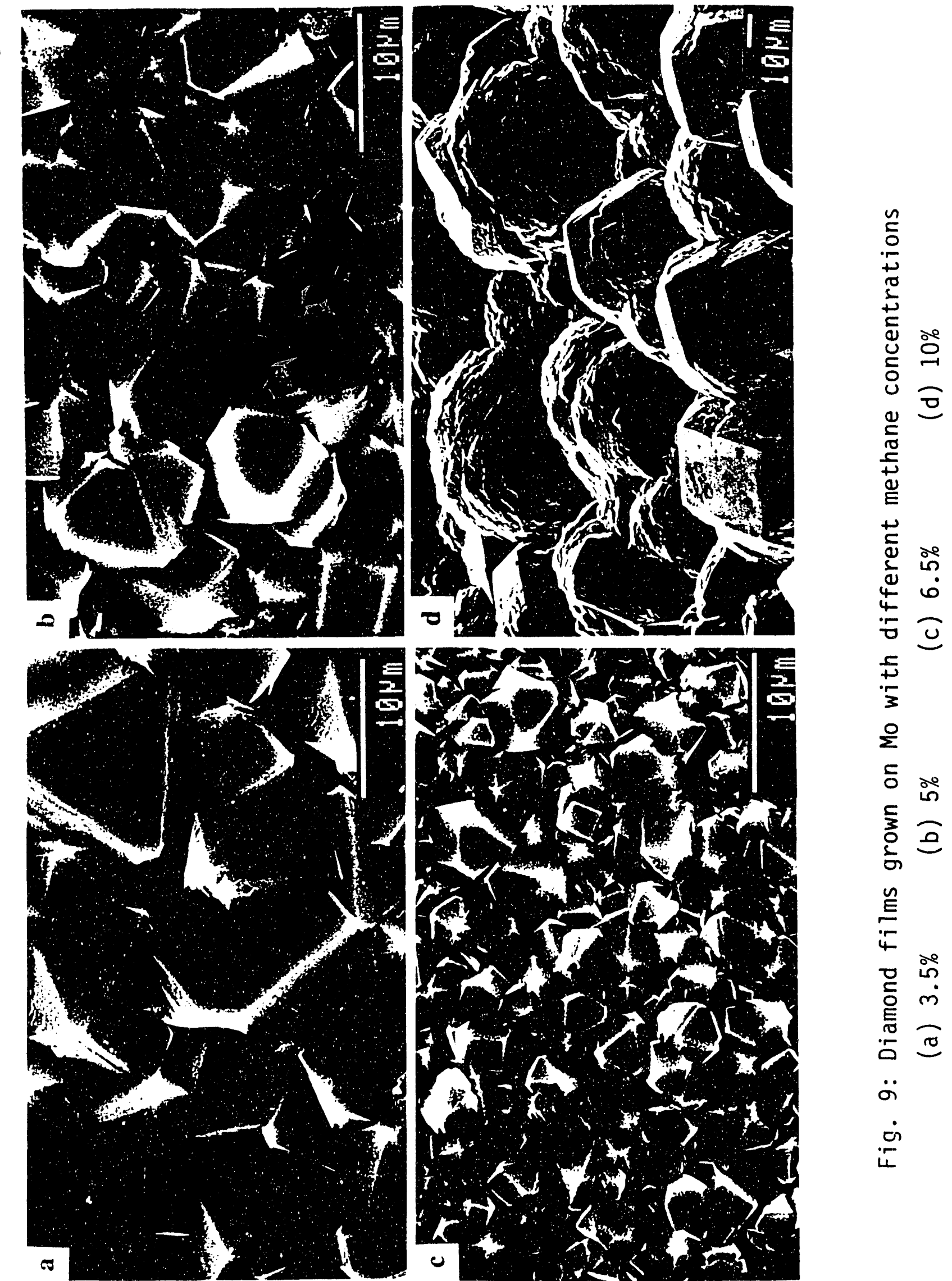



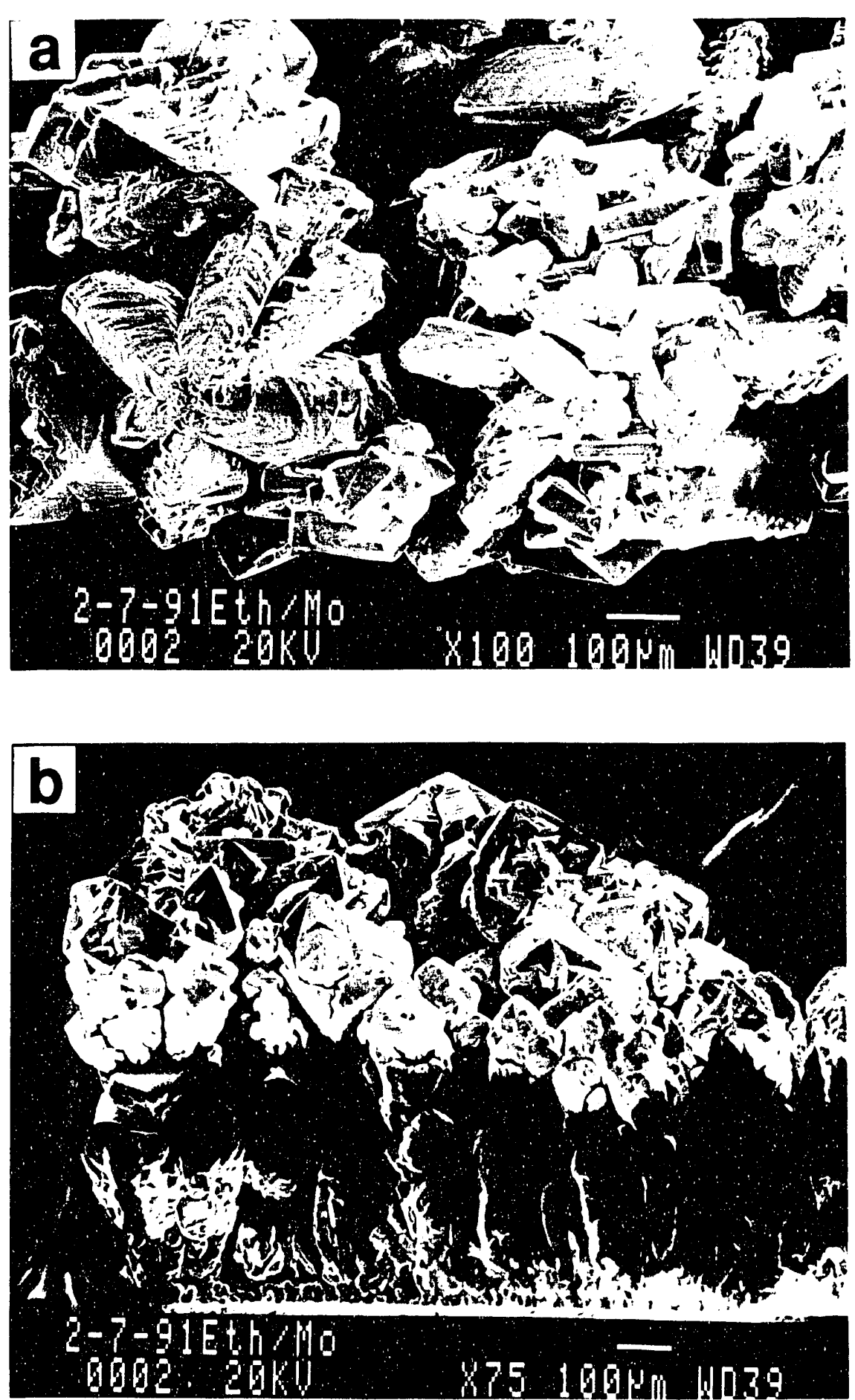

Fig. 10: Diamond films grown on Mo with ethanol as precursor 

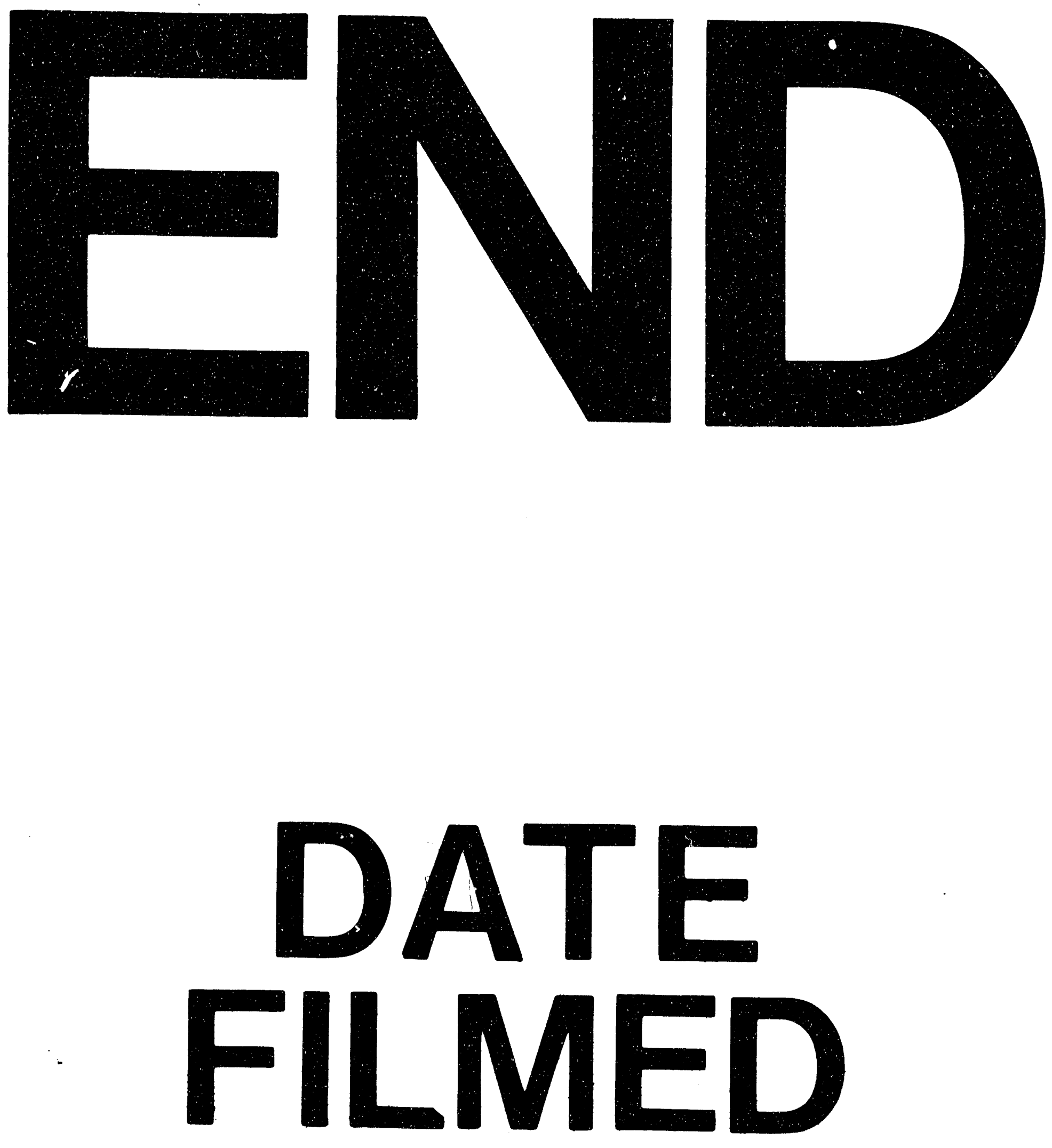

1

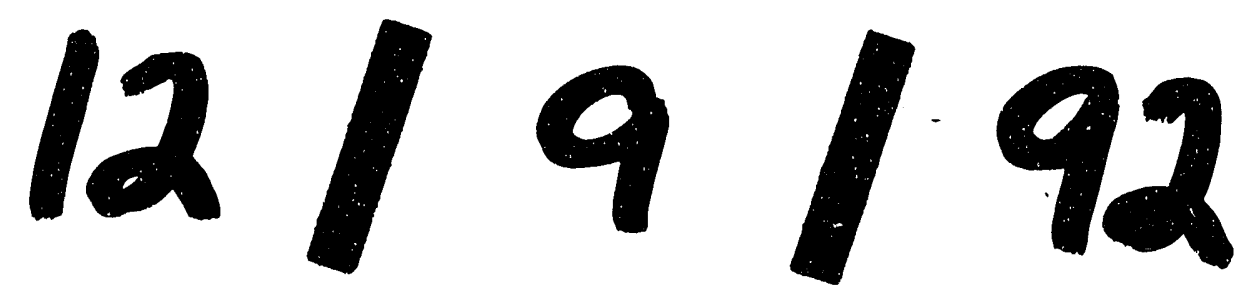


